\title{
Experimental demonstration of two-color x-ray free-electron-laser pulses via wakefield excitation
}

\author{
S. Bettoni® ${ }^{1,{ }^{*}}$ P. Craievich, ${ }^{1}$ A. Dax,${ }^{1}$ R. Ganter, ${ }^{1}$ M. W. Guetg $\odot,{ }^{2}$ M. Huppert $\odot,{ }^{1}$ F. Marcellini, ${ }^{1}$ \\ R. Neto Pestana, ${ }^{1}$ S. Reiche, ${ }^{1}$ E. Prat $\odot,{ }^{1}$ A. Trisorio $\odot,{ }^{1}$ C. Vicario, ${ }^{1}$ and A. A. Lutman ${ }^{3}$ \\ ${ }^{1}$ Paul Scherrer Institut, 5232 Villigen, Switzerland \\ ${ }^{2}$ Deutsches Elektronen-Synchrotron DESY, 22607 Hamburg, Germany \\ ${ }^{3}$ SLAC National Accelerator Laboratory, Menlo Park, California 94025, USA
}

(Received 27 November 2020; revised 26 February 2021; accepted 30 July 2021; published 16 August 2021)

\begin{abstract}
Several beam manipulation methods have been studied and experimentally demonstrated to generate $\mathrm{x}$-ray multipulses in free-electron-laser (FEL) facilities, advancing the fields of material science, molecular physics, and photochemistry by enabling x-ray pump/x-ray probe experiments. We have demonstrated a novel method to generate hard $\mathrm{x}$-ray multipulses using a hybrid compression scheme. Compared to other methods, this approach minimizes the time jitter among the FEL subpulses, produces large power FEL subpulses, is suitable for high repetition rate machines, since it does not imply the generation of losses, and it works at short and hard x-ray FEL wavelengths as well. All of these aspects make this approach attractive for the novel FEL facilities aiming to explore the hard x-ray region and to work at high repetition rate.
\end{abstract}

DOI: $10.1103 /$ PhysRevAccelBeams.24.082801

\section{INTRODUCTION}

$\mathrm{X}$-ray free-electron-laser (FEL) facilities operating in the so-called two-color mode allow the production of double $\mathrm{x}$-ray subpulses with control of their wavelength and subpulse delay to perform x-ray pump-probe experiments. Double subpulses have been used or proposed to be used to advance the fields of atomic, molecular and optical physics [1-3], to study magnetic skyrmion in probe/probe experiments [4], and to capture the dynamic of the explosion of water droplets [5].

After the initial demonstration [6,7], more schemes have been devised and experimentally tested [8-14] to increase the subpulse power and to improve the control over subpulse delay, duration, spectrum, and polarization, but there is not a single scheme outperforming all of the others in every aspect. The first approach is based on the split undulator scheme, theoretically predicted $[15,16]$ and experimentally demonstrated [6,8]. From the FEL resonance equation $\lambda_{R}=\frac{\lambda_{U}}{2 \gamma^{2}}\left(1+\frac{K^{2}}{2}\right)$ [17], where $\lambda_{R}$ is the wavelengths of the produced radiation, $\lambda_{U}$ the undulator period, $K$ the undulator parameter, and $\gamma$ the Lorentz factor, the undulator settings $\lambda_{U}$ and $K$, or the electron bunch $\gamma$ can be used to support various $\lambda_{R}$. Since the same electrons

\footnotetext{
*simona.bettoni@psi.ch
}

Published by the American Physical Society under the terms of the Creative Commons Attribution 4.0 International license. Further distribution of this work must maintain attribution to the author(s) and the published article's title, journal citation, and DOI. produce both photon subpulses, the power is limited to presaturation level for the first subpulse, otherwise the second subpulse is prevented to lase. This limitation has been improved by selectively controlling which bunch temporal slice lases in each undulator section, thus enabling tens of $G W$ postsaturation power and additional delay flexibility [11]. Such control has been demonstrated so far by a beam transverse tilt and subsequent bunch trajectory control [18-20], or by an optics time-dependent mismatch predicted in simulations [21], and experimentally demonstrated [22].

The setup of a two-color beam with the fresh-slice technique [11] requires a sufficiently short gain length to achieve saturation power in a fraction of the full undulator length. This is typically not achievable in the entire photon energy spectrum, failing in particular at shorter wavelengths. To produce fully saturated power at harder x rays, the twin-bunch [9] or two-buckets [14] schemes are preferable. In these schemes, two different bunches are extracted at the photoinjector, either illuminating the photocathode with two laser pulses using the pulsestacking technique [23] or with two independent drive lasers [24]. Both bunches are accelerated and compressed in the linac, and lase along the entire undulator line. The advantage of this approach is the efficient use of the undulator length, providing enough lasing intensity also at the shortest wavelengths. Drawbacks are the limited achievable color separation due to the bunch transport chromatic effects, and a more complicated setup impacting the whole machine from the cathode. Other approaches rely on the generation of current spikes along the bunch length 
using a slotted foil [25], sextupoles [26], generating a spatial tilt [18-20] or mismatch [21,22] along the bunch length. All these methods allow using the full undulator length, but may suffer from electron beam particle losses due to the transport of a bunch characterized by a highly degraded emittance, a transverse tilt, or optics mismatch along its length.

In this article we show the experimental generation of electron bunch multiple-current spikes and the associated production of multicolor hard x-ray FEL pulses by manipulating the longitudinal electron bunch phase space with an oscillating wakefield [27] induced along a passive structure installed upstream of the first bunch compressor. This method allows using the full undulator line and the majority of the electrons, it works for all the FEL wavelengths. These aspects make this method attractive especially for the hard $\mathrm{x}$-ray region. This approach does not imply the generation of losses, being ideal for high frequency FELs. The electron beam is manipulated only at a relatively high beam energy (few hundreds of $\mathrm{MeV}$ ), where the electron beam is already highly relativistic. This makes the beam degradation negligible and the setup time smaller compared to other approaches. Furthermore, this hybrid compression scheme, i.e., where the electron energy chirp is generated by the wakefield induced by the beam itself traveling through the passive structures and only partially by the rf, implies a smaller time jitter among the FEL subpulses compared to other methods, since the effect of the wakefield on the tail is self-synchronized with the head.

\section{METHOD}

Wakefield devices have been used for several applications in FEL facilities, including energy chirp control [28], phase space linearization [29,30], diagnostic purpose [31], and lasing control $[11,21,32]$. The method we present in this article has been explained in detail in [27], so here we only briefly recall it with the help of Fig. 1. The compression is controlled by adding an alternating sign energy chirp to that induced by the off-crest rf acceleration. This extra energy chirp is given by the wake potential excited by the beam itself passing through the passive structures. The wake potential may add or substract to the global energy chirp generated operating off crest the rf cavities. In case the periodicity of the wake potential is equal or smaller than half of the full bunch duration, two or more current spikes are generated using downstream magnetic chicanes. Each current spike lases along the entire undulator line, as in the twin-bunch scheme [9], but the setup is simpler, since it does not involve strong modifications at the lowest energy section of the machine. Like for the twin bunch scheme, the energy separation of the current spikes may be changed independently from the time delay by varying the phase of the rf structures downstream of the last compression stage, whereas the time delay adjustments require to manipulate the rf phases. In the case of the scheme discussed here the
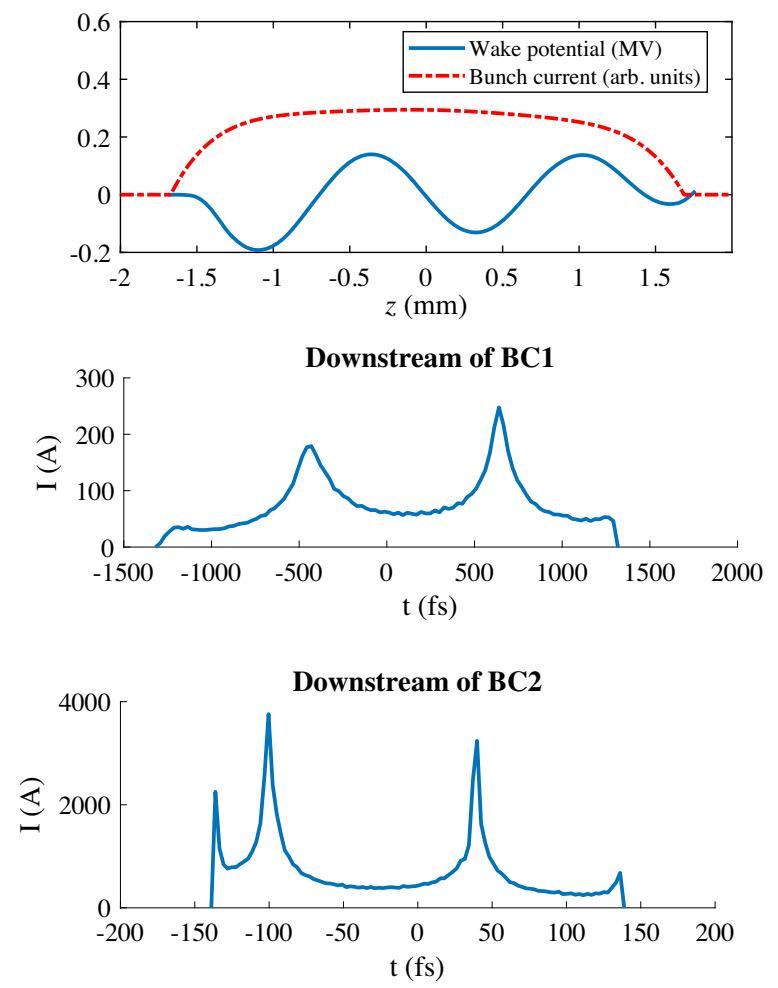

FIG. 1. Top plot: bunch longitudinal profile at the entrance of the passive structures, and energy loss (wake potential) along the electron beam induced by the bunch itself passing through the passive structures. The wake potential is computed as the convolution of the beam longitudinal profile and the wakefield point charge function of the passive structures. Middle plot: bunch longitudinal profile at the exit of the first bunch compressor. Bottom plot: bunch longitudinal profile expected downstream of the second bunch compressor.

time separation among the FEL subpulses may be varied also changing the periodicity of the oscillating wakefield, varying the gap of the passive structures. The obtained current spikes may have very short time durations and gain lengths at acceptable jitter due to the hybrid compression scheme, and are therefore perfect candidates to be combined with selective lasing control, or for multistage amplification [32,33].

\section{EXPERIMENT AT SWISSFEL}

We experimentally demonstrated the method proposed in [27] at the SwissFEL facility [34] at the Paul Scherrer Institute.

SwissFEL, whose schematic layout is shown in Fig. 2, has entered in regular user operation for hard x-ray radiation since the beginning of 2019 (pilot experiment from the end of 2017). The facility consists of several normal conducting accelerating sections (injector, linac 1, linac 2, and linac 3), which boost the beam energy from 7.1 $\mathrm{MeV}$ at the exit of the gun to the maximum design energy of $5.8 \mathrm{GeV}$ at the undulator line. At the 


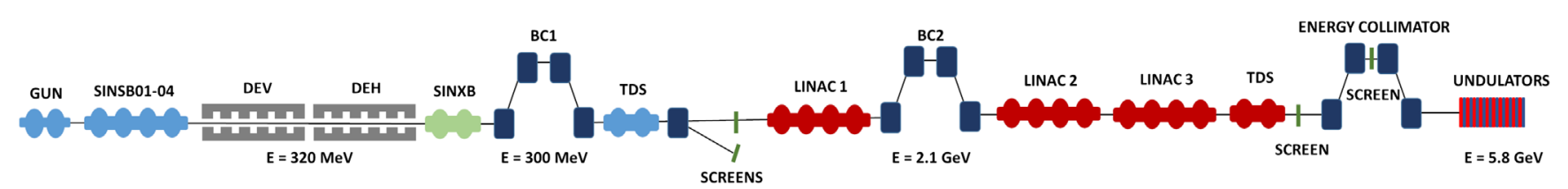

FIG. 2. SwissFEL schematic layout (not to scale). The hard x-ray branch used for the measurements described in this article is shown. Different colors indicate different frequencies of the rf structures. Light blue corresponds to S-band (gun length of $0.15 \mathrm{~m}$, and accelerating structures with a total length of $32 \mathrm{~m}$ length for four structures), green to X-band (total length of $1.5 \mathrm{~m}$ length for two structures), and red to C-Band (total length of $208 \mathrm{~m}$ distributed among the 26 structures of the three linacs). The length of BC1 and BC2 are 14 and $17 \mathrm{~m}$, respectively. The transverse deflecting structures (TDS) used to characterize the slice beam parameters are also shown. The total length of the machine is about $730 \mathrm{~m}$ from the gun to the undulator exit. The passive structures, DEH and DEV (total length of $2 \mathrm{~m}$ ), are also indicated.

photoinjector the $200 \mathrm{pC}$ charge electron bunch is extracted from a $\mathrm{Cs}_{2}$ Te photocathode illuminated by a $260 \mathrm{~nm}, 10 \mathrm{ps}$ FWHM flattop longitudinal profile (initial SwissFEL design). The design $3 \mathrm{kA}$ peak current at the undulator entrance from the $20 \mathrm{~A}$ at the exit of the injector is obtained using in cascade two 4-dipoles magnetic chicanes, $\mathrm{BC} 1$ and $\mathrm{BC} 2$, at an energy of $300 \mathrm{MeV}$ and $2.1 \mathrm{GeV}$, respectively. The energy chirp to achieve the compression at $\mathrm{BC} 1$ is provided by the last two of the four S-band structures (SINSB03-04). The chirp for the second compression stage is mainly induced by the linac 1 structures. A X-band cavity (SINXB) installed at the injector is used to linearize the bunch longitudinal phase space for both compression stages. The linac 2 and linac 3 rf structures operate on crest solely to boost the beam energy up to the design value. The undulator line is equipped with 13 in-vacuum variable gap undulators, to generate FEL radiation in a wavelength ranging from 0.1 to $0.7 \mathrm{~nm}$. More details about SwissFEL can be found in [34].

We installed two pairs of $1 \mathrm{~m}$ each long aluminum corrugated parallel plates (DEV and DEH in Fig. 2) between the last S-band structure and SINXB, at a location where the beam has an energy of $320 \mathrm{MeV}$. At this location the beam excites the oscillating longitudinal wakefield. The two structures, DEV and DEH, are orthogonally oriented to compensate for the quadrupole wakefield [35], and their gap and center position are remotely controlled between 0.7 and $30 \mathrm{~mm}$ (the latter which corresponds to no effect on the beam) and 0 and $15 \mathrm{~mm}$, respectively. The periodicity of the corrugation is $0.5 \mathrm{~mm}$, the space between the corrugations is $0.25 \mathrm{~mm}$, and its depth is $0.1 \mathrm{~mm}$. The geometry of the corrugation is optimized to generate the correct number of wakefield modulations inside the full bunch duration, as described in [27].

We distinguish the global chirp, generated by going off crest in the rf structures, from the local chirp, impinged by the longitudinal wakefield due the corrugated plates, and the total chirp, given by the sum of the two. We adjusted the global chirp to moderately compress the beam by a factor 3 (from 20 to about $60 \mathrm{~A}$ ), then we obtained about $180 \mathrm{~A}$ current spikes closing DEV and DEH down to $2 \mathrm{~mm}$ gap inducing the local chirp. The change of the SINSB03-04 phase and the moderate variation of the SINXB amplitude $(15 \%)$ are the only necessary modifications to the machine settings, and they occur at an energy where the electron bunch is already heavily relativistic. After being compressed at $\mathrm{BC} 1$ and $\mathrm{BC} 2$, the beam features the expected current spikes separated in time and energy, as shown in Fig. 3.

There is a vast number of combinations of global and local chirps giving the same target peak current at $\mathrm{BC} 1$. We adjusted the set point of the injector optimizing the quality of the compressed beam (slice and projected emittance and optics mismatch along the bunch duration), and the ratio of the current peak at the current spikes location and in between. The first condition maximizes the FEL intensity, whereas the latter guarantees FEL spectra with a region characterized by zero or negligible lasing intensity between the pump and probe subpulse. Finally, we selected the working point of linac 1 , linac 2 , and linac 3 to obtain the desired peak current and energy separation in between the current spikes.
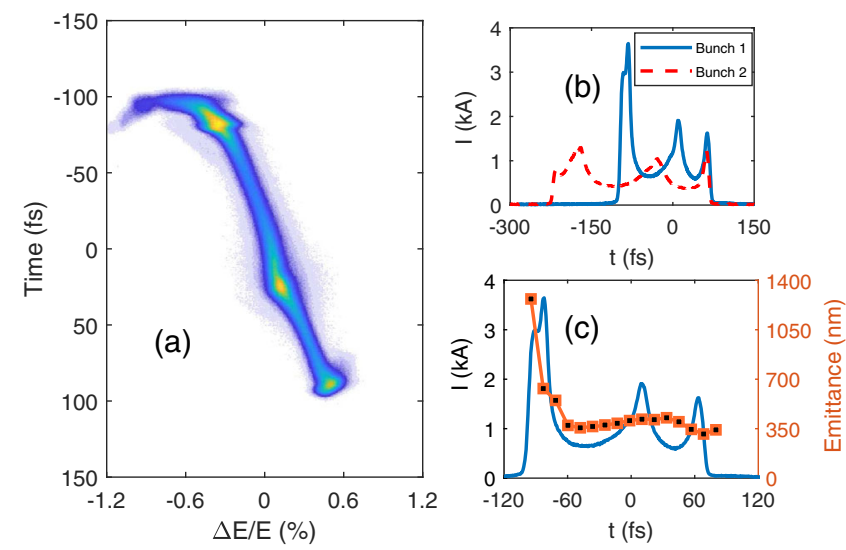

FIG. 3. Typical electron bunch characterized at the energy collimator. (a) Bunch longitudinal phase space. (b) Beam longitudinal current profiles. We repeated the measurements with different time separations among the current spikes (bunch 2). This has been obtained varying the off-crest phase of the linac $1 \mathrm{rf}$ structures. (c) Normalized slice emittance and beam current longitudinal profile referring to the Bunch 1 case reported in (b). 


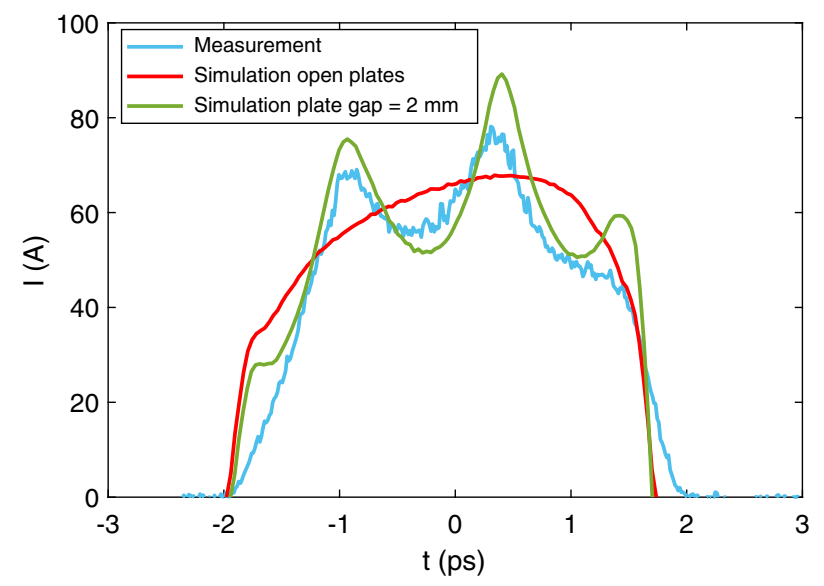

FIG. 4. Measured bunch longitudinal current profile compared with the results of the LiTRack simulations obtained using the wakefield computed using ECHO corresponding to the case of one plate closed at $2 \mathrm{~mm}$ gap. The simulated current profile corresponding to the open plates is also reported. The parameters of the structure are stated in the text.

We compared the results from several codes to compute the longitudinal wakefield generated by the passive structures installed at SwissFEL. We found a very good agreement between the results obtained using ЕCHO [36] and CST STUDIO [37]. We used LiTRack [38] and the wakefield obtained by ECHO to simulate the dynamics of the electron bunch traveling along one of the passive structures. Figure 4 shows the comparison of the beam longitudinal profile measured downstream of the first bunch compressor with the simulation results obtained with LiTRack with the wakefields calculated by ECHO for a single structure with a gap of $2 \mathrm{~mm}$. Figure 4 shows the comparison of the results of the LiTRack simulations with the measurements. We found quite a good agreement of the periodicity of the current spikes, but a discrepancy on the amplitude. This effect may be due to an uncertainty on the value of the gap of the passive structures, the charge reading, or some other parameters of the beam like the initial pulse duration. The observed small discrepancy did not preclude the application of the method. During the experiment we slightly reduced the gap of the structures (few hundreds of $\mu \mathrm{m}$ over the expected $2 \mathrm{~mm}$ ) to compensate for it.

We had the possibility to generate up to three current spikes using a laser 10\% longer than in simulations, and further closing the passive structures. This setup allows for the generation of three-color spectra, even if in our case the third spike (at the tail of the bunch) was lasing only for a small fraction of the pulses. This may be due to some suboptimal trajectory along the passive structures, which starts to be important going towards smaller gaps and the tail of the beam.

In Fig. 3 we show the beam at the entrance of the undulator line, which generates multicolor FEL spectra like those shown in Fig. 5. The FEL pulse energy is measured

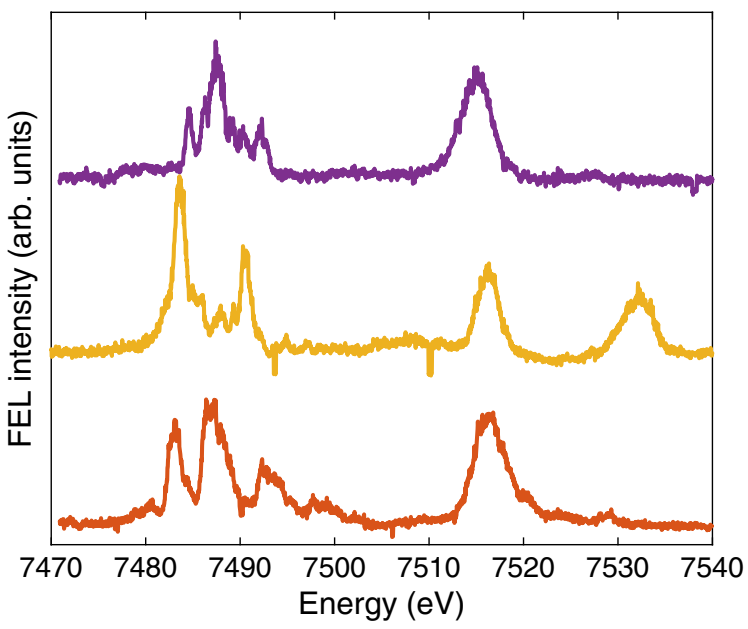

FIG. 5. Some examples of the multicolor FEL spectra measured by the single-shot photon spectrometer [39] installed at the end of the undulator line. For completeness we include one of the threecolor spectra (middle spectrum).

by a gas-based photon-intensity monitor [39]. In the case of the two-color mode we obtained a pulse energy of a few tens of $\mu \mathrm{J}$ with a maximum of about $50 \mu \mathrm{J}$ starting from about $140 \mu \mathrm{J}$ in the SASE mode. Similarly to other methods based on the inhibition of the lasing along a portion of the bunch duration, the FEL intensity is reduced when the two colors are generated. The length of the single FEL subpulse may be determined by the number of spikes in the energy distribution of the FEL subpulses from a statistical analysis, as described in [40]. According to this analysis a single FEL spike of the energy spectrum corresponds to a FEL rms pulse length of the order of a few fs or less, well below the factor 3 shorter pulse length. This implies that the power of the radiated FEL is increased for the two-color setup also if the total intensity is reduced.

An important aspect of any two-color scheme is the tunability of different properties of the FEL subpulses. We experimentally demonstrated the possibility of changing the relative intensity, the time and the energy separation among the FEL subpulses.

There are three ways to vary the relative intensity of the FEL subpulses: modifying the SINXB amplitude, generating optics mismatch, or introducing a time-dependent tilt along the bunch. The first method has a limited tunability, because one of the two peaks may suffer a possible beam emittance degradation due to a nonlinear compression. We measured a slice emittance degradation of a factor 2 with $17 \%$ variation of the SINXB amplitude from the optimal value. The latter option appears to be much more simple than the second one, because it relies on a modification of the setting of a single element relatively close to the undulator line. We introduced a tilt along the bunch varying by a maximum of $2 \%$ the strength of a quadrupole located at a dispersive section in the energy collimator. Figure 6 shows the measured FEL average spectra over 1000 

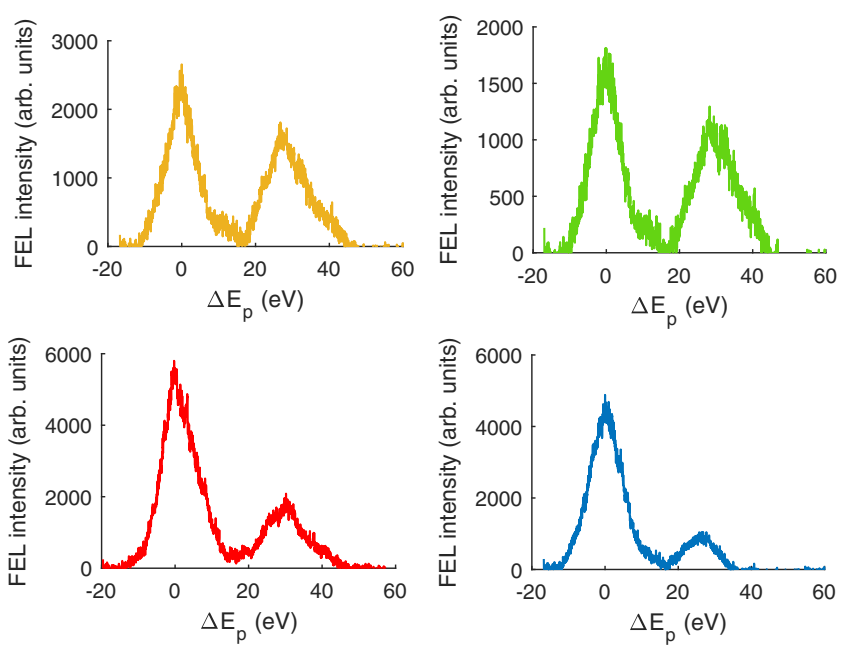

FIG. 6. Average photon spectra obtained measuring the photon intensity downstream of a monochromator scanning its energy setpoint, $E_{p}$. Each subplot corresponds to a different quadrupole strength (maximum variation of $2 \%$ ). The rms values of the lower and higher energy color are $8 \pm 1$ and $6.5 \pm 0.5 \mathrm{eV}$, respectively. The $0 \mathrm{eV}$ photon energy in the abscissa corresponds to $7.5 \mathrm{keV}$.

consecutive shots. In this way we varied the intensity ratio among the two FEL subpulses from a ratio of 1.5 up to 4.7.

Two options are available to tune the time separation between the FEL subpulses: changing the gap of the corrugated structures, or modifying the global chirp. We used the latter option, common to other methods. Figure 3(b) shows the current profile measured for two different global chirps. In this particular case, we measured a change of the time separation of $40 \mathrm{fs}$. Like for other methods, the time delay is coupled with the energy separation, so after the first has been adjusted, the latter one must be tuned. The time separation can go up to more than 100 fs [27] changing the gap of the passive structures.

The energy separation between the FEL subpulses may be adjusted independently from the time delay changing the phase of rf structures downstream of the last compression stage, i.e., linac 2 and linac 3, since the energy collimator operates with $R_{56}=0 \mathrm{~m}$, i.e., in isochronous mode. Compared to what was described in [27], we enhanced the energy separation between the subpulses setting the compression at the exit of $\mathrm{BC} 2$ to overcompression. In this configuration the linac 2 and linac 3 longitudinal wakefields add to the beam energy chirp, and, consequently, the energy separation among the current spikes is enhanced. This setup is routinely used to obtain large-bandwidth FEL spectra [41]. We measured about $2 \%$ energy separation between the FEL subpulses at $7 \mathrm{keV}$ photon energy. To perform an experiment with large (small) energy separation between the FEL subpulses, the beam is set up in overcompression (undercompression) mode at the exit of $\mathrm{BC} 2$, and the linac 2 and linac 3 rf phases are changed only to fine-tune the energy separation. We experimentally

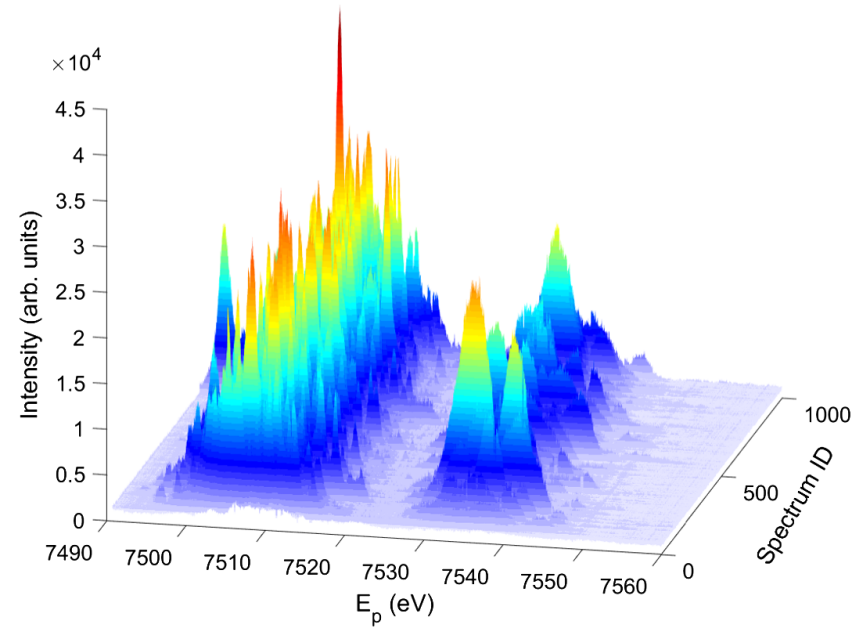

FIG. 7. 1000 consecutive spectra measured by the photon single-shot spectrometer. The number of spikes and therefore the duration of the high energy color is minimized.

demonstrated a variation of the energy separation from $0.4 \%$ up to $2 \%$ at $7 \mathrm{keV}$ photon energy at SwissFEL using this strategy. These values are specific of each machine. A larger energy gain downstream of the last bunch compressor or stronger wakefields of the rf structures would allow to further enhance the maximum energy separation.

Figure 7 shows 1000 consecutive single-shot FEL spectra. We took the series of spectra changing the number of spikes of the high energy color, which, in the case reported in Fig. 7, is expected to be much shorter than the low energy color subpulse.

\section{CONCLUSIONS}

We have experimentally demonstrated a method to generate a multispike current profile within an electron bunch able to lase in the soft and in the hard x-ray wavelength region. The current spikes are generated using an oscillating longitudinal wakefield excited by the electron beam itself passing through dedicated passive structures installed at a relatively high beam energy. We showed the tunability of the properties of the FEL subpulses, in terms of separation in time and energy, relative amplitude of the FEL subpulses, and independent control of the length of the single FEL subpulse as well. Compared to other approaches used to obtain two-color FEL pulses, this method is extremely promising to generate large power FEL twocolor subpulses, since it does not imply a massive modification of the machine parameters in the low-energy section of the accelerator, it allows using the entire undulator line, and it does not imply any beam degradation to inhibit the lasing along a portion along the electron bunch duration. The method is therefore particularly attracting for the new FEL machines, aiming to explore the hard x-ray region around the $20 \mathrm{keV}$, where other approaches would be very inefficient. The scheme we 
demonstrated is also interesting for the new high repetition rate machines, since it does not imply charge losses along the accelerator or along the undulator line.

Furthermore, we have demonstrated a hybrid approach to control the bunch compression employing both if and passive structures. This represents a valid way to improve the FEL stability not only of the two-color setup, but for other operation modes. The same method may be extended to any other machine to improve the time jitter of the compressed beam.

\section{ACKNOWLEDGMENTS}

The authors are grateful to P. Heimgartner, H. Joehri, and N. Kivel for the excellent work building and bringing into operation the passive structures, to C. Cirelli, P. Juranic, Y. Arbelo, and C. Arrell for supporting us to set up the photon diagnostics, E. Ferrari for the fruitful discussions, and H. H. Braun and T. Schietinger for the proofreading of the manuscript. A. A. Lutman acknowledges support by DOE Contract No. DE-AC02-76SF00515.

[1] A. Picon et al., Nat. Commun. 7, 6369 (2016).

[2] N. Berrah et al., Nat. Phys. 15, 1279 (2019).

[3] K. R. Ferguson et al., Sci. Adv. 2, e1500837 (2016).

[4] M. H. Seaberg et al., Phys. Rev. Lett. 119, 067403 (2017).

[5] C. A. Stan et al., Nat. Phys. 12, 966 (2016).

[6] A. A. Lutman, R. Coffee, Y. Ding, Z. Huang, J. Krzywinski, T. Maxwell, M. Messerschmidt, and H.-D. Nuhn, Phys. Rev. Lett. 110, 134801 (2013).

[7] D. A. Jaroszynski, R. Prazeres, F. Glotin, and J. M. Ortega, Phys. Rev. Lett. 72, 2387 (1994).

[8] T. Hara et al., Nat. Commun. 4, 2919 (2013).

[9] A. Marinelli et al., Nat. Commun. 6, 6369 (2015).

[10] A. A. Lutman et al., Phys. Rev. Lett. 113, 254801 (2014).

[11] A. A. Lutman et al., Nat. Photonics 10, 745 (2016).

[12] A. A. Lutman et al., Nat. Photonics 10, 468 (2016).

[13] A. Marinelli et al., in Proceedings of the International Particle Accelerator Conference (IPAC2016), Busan, Korea (JACoW, Geneva, Switzerland, 2016).

[14] F.-J. Decker, K. Bane, W. Colocho, A. Lutman, and J. Sheppard, in Proceedings of the International Free Electron Laser Conference (FEL17), Santa Fe, NM, 2017, International Free Electron Laser Conference No. 38 (JACoW, Geneva, Switzerland, 2018), pp. 288-291, https://doi.org/10.18429/JACoW-FEL2017-TUP023.

[15] G. Geloni, V. Kocharyan, and E. Saldin, DESY Technical Report No. 10-004, 2010.

[16] G. Geloni, V. Kocharyan, and E. Saldin, DESY Technical Report No. 10-006, 2010.

[17] R. Bonifacio, C. Pellegrini, and L. Narducci, Opt. Commun. 50, 373 (1984).

[18] S. Reiche and E. Prat, J. Synchrotron Radiat. 23, 869 (2016).
[19] E. Prat, S. Bettoni, and S. Reiche, Nucl. Instrum. Methods Phys. Res., Sect. A 865, 1 (2017).

[20] M. W. Guetg, A. A. Lutman, Y. Ding, T. J. Maxwell, and Z. Huang, Phys. Rev. Lett. 120, 264802 (2018).

[21] W. Qin, Y. Ding, A. A. Lutman, and Y.-C. Chao, Phys. Rev. Accel. Beams 20, 090701 (2017).

[22] Y.-C. Chao, W. Qin, Y. Ding, A. A. Lutman, and T. Maxwell, Phys. Rev. Lett. 121, 064802 (2018).

[23] V. Petrillo et al., Phys. Rev. Lett. 111, 114802 (2013).

[24] F.-J. Decker et al., in Proceedings of the 10th International Particle Accelerator Conference (IPAC'19), Melbourne, Australia, 2019, International Particle Accelerator Conference No. 10 (JACoW Publishing, Geneva, Switzerland, 2019), pp. 1859-1862, https://doi.org/10.18429/JACoW-IPAC2019-TUPRB086.

[25] P. Emma, K. Bane, M. Cornacchia, Z. Huang, H. Schlarb, G. Stupakov, and D. Walz, Phys. Rev. Lett. 92, 074801 (2004).

[26] P. Dijkstal, A. Malyzhenkov, S. Reiche, and E. Prat, Phys. Rev. Accel. Beams 23, 030703 (2020).

[27] S. Bettoni, E. Prat, and S. Reiche, Phys. Rev. Accel. Beams 19, 050702 (2016).

[28] P. Emma et al., Phys. Rev. Lett. 112, 034801 (2014).

[29] P. Craievich, Phys. Rev. ST Accel. Beams 13, 034401 (2010).

[30] G. Penco et al., Phys. Rev. Lett. 119, 184802 (2017).

[31] S. Bettoni, P. Craievich, A. A. Lutman, and M. Pedrozzi, Phys. Rev. Accel. Beams 19, 021304 (2016).

[32] E. Prat, F. Löhl, and S. Reiche, Phys. Rev. ST Accel. Beams 18, 100701 (2015).

[33] A. A. Lutman, M. W. Guetg, T. J. Maxwell, J. P. MacArthur, Y. Ding, C. Emma, J. Krzywinski, A. Marinelli, and Z. Huang, Phys. Rev. Lett. 120, 264801 (2018).

[34] C. J. Milne et al., Appl. Sci. 7, 720 (2017).

[35] Z. Zhang, K. Bane, Y. Ding, Z. Huang, R. Iverson, T. Maxwell, G. Stupakov, and L. Wang, Phys. Rev. ST Accel. Beams 18, 010702 (2015).

[36] I. Zagorodnov and T. Weiland, J. Comput. Phys. 207, 69 (2005).

[37] CST, CST Microwave Studio Advanced Topics, CSTComputer Simulation Technology Technical Report, 2008, http:// www.cst.com.

[38] P. E. K. L. Bane, https://www.osti.gov/biblio/839868 (2005).

[39] P. Juranić, J. Rehanek, C. A. Arrell, C. Pradervand, R. Ischebeck, C. Erny, P. Heimgartner, I. Gorgisyan, V. Thominet, K. Tiedtke, A. Sorokin, R. Follath, M. Makita, G. Seniutinas, C. David, C. J. Milne, H. Lemke, M. Radovic, C. P. Hauri, and L. Patthey, J. Synchrotron Radiat. 25, 1238 (2018).

[40] A. A. Lutman, Y. Ding, Y. Feng, Z. Huang, M. Messerschmidt, J. Wu, and J. Krzywinski, Phys. Rev. ST Accel. Beams 15, 030705 (2012).

[41] E. Prat, P. Dijkstal, E. Ferrari, and S. Reiche, Phys. Rev. Lett. 124, 074801 (2020). 